\title{
Charge Trapping Phenomenon in Al-Filled Epoxy Nanocomposite Materials: Current Status and Challenges for Insulation Structures
}

\author{
Chillu Naresh ${ }^{1}$, Rengaswamy Jayaganthan ${ }^{2}$, Ramanujam Sarathi ${ }^{1 *}$ and Michael G. Danikas ${ }^{3}$ \\ ${ }^{1}$ Department of Electrical Engineering, Indian Institute of Technology Madras, Chennai 600 036, India. \\ ${ }^{2}$ Department of Engineering Design, Indian Institute of Technology Madras, Chennai 600 036, India. \\ ${ }^{3}$ Department of Electrical and Computer Engineering, Democritus University of Thrace, 67100 Xanthi, Greece.
}

Received 19 April 2021; Accepted 25 June 2021

\begin{abstract}
In the present work, a criticalreview is made on methods of surface potential measurements adopted on various polymeric materials, mechanisms of charge decay and trap distribution associated with the respective materials. Surface potential measurements and trapping characteristics of polymeric materials reported in the literature are critically assessed to provide glimpses of its growing demand for the improved performance of insulation structures in power system networks. The present review ensues with the preamble of the importance of corona discharge of various insulators and subsequently it dwells on particularly on the development of polymeric insulating materials in the high voltage engineering. The importance and method of surface charge measurements of polymeric insulating materials are presented. The needle plane configuration setup employed to investigate the surface charge decay characteristics are described. It is chosen due to its non-contact, easy to use and adaptability to desired experimental conditions. After the charging process, the various routes of charge decay and different detrapping processes involved in the charge neutralization, along with their importance on underlying experimental conditions, are elaborated further. The development of the different type of traps in the material and their role in accumulating the charge in the vicinity of the defective states are systematically evaluated. Various important parameters such as initial potential, decay rate, trap depth and trap charge density are used in elucidating the charge trapping behaviour of the polymeric materials. The variation in the surface potential as well as the respective trap distribution due to the Aluminium (Al) nanofiller addition, under different voltage profiles and different irradiated environments are elucidated. Controlled addition of Al nanoparticles to base epoxy matric has shown an increased decay rate. On the other hand, increased rise time of the applied transient voltage has resulted in the deep trap sites in the nanocomposite material. The induced trap energy states due to the gamma irradiation in the epoxy matrix are nullified by the addition of $\mathrm{Al}$ filler content.
\end{abstract}

Keywords: trap characteristics, surface potential, nanocomposites, gamma irradiation, charge decay, neutralization, corona discharge

\section{Introduction}

Surface and/or internal charging of materials occur when a sufficiently high electric field is exerted from the electrodes (sharp protrusions, wire or a plate). The electrons in the surrounding ambience of emitting electrode act as ionizing agents, upon being excited by the electric field, the energy is transferred to the gas molecules, and therefore the avalanche of energized molecules trigger the corona discharge [1]. The trajectory of ions is dictated by the electric field, and the corresponding discharge, in general, possesses the same polarity as that of the high voltage electrode. Further, the discharge is controllable because the increase in the electric field between electrodes increases the corona current.

Electro-optics, for instance, xerography in specific, corona discharge is being employed to charge the photoconductor surface uniformly at the initial stage of photocopying and printing applications [2]-[4]. Maintaining a constant surface potential despite the charge transport mechanism, is achieved through the introduction of trappings till the charged particles are removed from the photoconductor surface [3]. The corona discharge is used in

*E-mail address: rsarathi@iitm.ac.in

ISSN: 1791-2377 @ 2021 School of Science, IHU. All rights reserved.

doi:10.25103/iestr.143.20 electrostatic precipitator applications in collecting the contaminated charged particles in an efficient way from the industrial gases [5], [6]. In the fabrication of electrets, corona discharge is proven to be the best method in distributing the charge uniformly and maintaining the charge stability for a long time over the surface in comparison with other techniques such as liquid contact and electron beam method, in generating the permanent electric field in the solid dielectric material [7]-[9]. Large scale production of reliable and low-cost piezoelectric transducers for biomedical instrumentation is now possible through the surface discharge technique, wherein the method is mainly used for polarizing the polymeric material in obtaining the required electric field without the major breakdown of underlying material [10][12]. Upon interaction with the charged particle, the surface of the material experiences various reactions such as crosslinking, degradation and radiation-induced conductivity in the irradiated nuclear environment [13]. Thus, gamma irradiated epoxy resin under repetitive pulse voltage has shown a significant variation in trap distribution characteristics [14]. Corona discharge is an elegant way to analyse the effect of cosmic rays on the dielectric materials that are used to evaluate the conductance and trapping performance of the material [15]. Further, corona discharge is used in indoor air cleaners, treatment of polymeric surfaces, 
production of ozone, and destruction of gaseous contaminants [16].

Apart from the applications mentioned above, corona discharge is also used to evaluate the surface trapping characteristics of the insulating materials used in high voltage and power transportation and distribution networks [17], [18]. Effective utilization of surface discharge technique yields fruitful results, particularly, the electrets, wherein the charge build-up, stabilization and trapping into the material for a long time is an essential requirement. On the other hand, uniform charge distribution and dissipation at a rapid rate and at regular intervals of time over the photoconductive film is one of the essential requirements in electrophotography [19]. Whereas, a least surface charge accumulation and quick charge dissipation are the desired parameters in obstructing the space charge formation in bulk, thereby refraining the possible damage to the network components in the near future in case of the electrical insulation design application. With the above description of different applications in which corona discharge finds its prominence, it is evident from the perspective of the electrical insulation that the corona discharge is not always a threat for the insulation as long as the least possible charge accumulation during the network operation and rapid charge dissipation upon removal of electrical stresses are maintained [20], [21].

The present review is mainly focused on surface charge decay characteristics of polymeric insulating materials utilized in various high voltage applications. Polymeric materials are now finding their prominence as the insulating material in long-distance electrical power transmission systems due to their advantageous technical aspects over the conventionally used ceramic (glass and porcelain) based insulation [22]-[24]. Laboratory and onsite test performance of silicone rubber composites have shown improved mechanical, thermal and dielectric properties, besides their inherent advantages of hydrophobic performance, lightweight and vandal resistance [25]-[28]. The surface of the insulator is primarily exposed to the environment where the strongly localized constraints such as uncontrolled weather conditions as well as the places that are in the vicinity of the pollution sources (cities, sea, plants etc.) dictate the performance of insulating material during the power system operation. Insulators in the proximity of coastal areas are more prone to the formation of conductive channels over their surface due to the accumulation of sea salt [29], [30]. Though the contamination layer over the surface does not cause a severe problem in dry conditions, it is a considerably a serious threat to the entire power system network in the presence of wetting agents such as humidity, fog, dew, drizzling and misty conditions [31]. Sooner or later, the bridging causes the leakage current to flow from high voltage end to ground electrode, and thereafter, the dry bands are formed over the insulating material surface due to the intense heating preceded by the water evaporation along the pollution initiated conductive channels [30]-[33]. The network of partial discharges across the dry band leads to the occurrence of complete flashover, and hence the inevitable premature failure of the power system can result [34], [35].

Further, the glass fiber reinforced polymeric materials are preferred in construction, aviation and electronics industries due to their high strength, stiffness and resistance to harsh environments [36]-[38]. Nevertheless, these materials also experience surface discharges in space due to uncontrolled irradiation levels, satellite anomalies, etc. [39], [40]. Epoxy is a widely used insulating material for superconducting magnets in controllable nuclear fusion research. The reactions such as crosslinking and degradation occur to the insulation due to prolonged usage in the electron beam and gamma irradiation environment [41], [42]. The non-conductive insulating materials play an equally responsible role as that of the conducting materials in power transmission and distribution networks. Epoxy resin is one such thermosetting polymeric insulating material that protects the electrical and power electronic components from short circuits, moisture and dust accumulation besides providing structural support for the live components. Specifically, the epoxy insulation is used as a housing material for superconducting fault current limiters in the power system network [43]-[45]. The network components such as cables, transformers, overhead lines and switchgear units are designed to withstand the fault currents typically higher than the normal load currents for a negligible period of time. In the advent of prolonged fault currents, the network components experience increased fault levels and exceeding the network component ratings can lead to catastrophic failure of the network if the proper remedies are not incorporated. Fault current limiter (FLC) is one such addition to the network with the dynamic impedance characteristics, which can save the power system by offering negligible impedance during the normal load currents and offering a rapid rise in impedance, on the other hand, during the presence of a fault or high currents for longer periods of time. The insulation in the FLC device is subjected to a rapid rise in the thermal and electrical stresses during the faulty events along with the quenching effects. The breakdown characteristics of the insulation under liquid nitrogen $\left(\mathrm{LN}_{2}\right)$ is greatly influenced by the volume defects, rise in temperature and creepage discharge properties under the cryogenic environment [43], [46]. Recently, epoxy filled with titania and alumina nanoparticles has shown better dielectric characteristics over the epoxy material alone. Further, the addition of silica nanoparticles has improved the Alternating current (AC) breakdown as well as partial discharge inception voltage characteristics over the base polymeric material [46], [47]. In addition, the epoxy polymeric materials are widely used insulating structures for the various power network components such as gas insulated substation (GIS) spacers, cable joints, dry-type transformers, post type insulators and as a support structure for bus bar and breaker in switchgear [48][51].

Tam introduced the trapping and detrapping through the demarcation energy model for semiconducting and insulating materials [52], [53]. Watson has adopted the model and obtained the trapping parameters by the application of the electron beam charging method [54], [55]. The surface demarcation energy model was introduced by Molinie et al., and it was used extensively in estimating the trap characteristics of various insulating materials during the potential decay [18], [19], [56]-[59]. Gubanski and his coworkers have worked extensively on measuring and modelling the surface potential measurements of various polymeric materials, which are in service to analyze the surface performance analysis of the respective materials [26], [60]-[63]. Two-level surface charge trapping process at various defects possessing different energy levels are investigated by Chen et al. through isothermal surface potential decay studies [21], [64]. Elaborative research work on surface potential decay measurements and corresponding trap characteristics of various polymeric materials under different ageing condition, nanofiller additions, thermal and mechanical stresses, and surface functionalization etc. are studied by Du et al. [65]-[74]. 
The magnitude of the charge accumulation under the High voltage direct current (HVDC) stress is strongly related to the unavoidable defective states in the insulation, which further lead to the charge trapping phenomenon [75]. Here, the HVDC is the most effective and economical means of power transfer technology providing grid interconnection and more controllable power transfer over a long distance while maintaining network quality and stability, besides offering lower losses in comparison with the AC transmission system [76].

With the significance of corona discharge known in various applications, it's usage in insulation point of view in high voltage applications is further explored through a needle plane configuration test setup, a well adopted method for obtaining the surface potential measurements under different voltage profiles, temperature and ageing conditions. Rest of the paper deals with the corona discharge and surface potential measurement experimental setup, the process of charge injection and trapping, mechanisms of charge neutralizations, possible routes of charge decay, surface charge measurements through Kelvin probe method, and trap distribution analysis through the surface potential measurement parameters such as mean life time and trap depth. The three case studies are discussed in order to examine the influence of nanoparticle addition, variation in voltage profile and gamma ageing effect on surface potential decay and trap distribution characteristics of Al filled epoxy nanocomposite materials.

\section{Experimental setup}

The defective states nearing the insulating material surface are more vulnerable to charge accumulation due to the sustained electric field during the electrical network operation under the Direct current (DC) voltage [75]. Nevertheless, the polymeric material surface is prone to charge accumulation mainly due to surface discharges, triboelectric effect, polarization, and charge migration etc. Extended periods of operation under the high fields can distort the structure of local electric fields within the material [77]. The built over stress as a result of electric field enhancement at the defective states will show a deteriorative effect, at worst, breakdown of the resultant insulating material, over a long term operation. Thus, the surface potential can compel the electric fields high enough to cause the surface discharge activity or on the charge being trapped in localized states can cause enhancement in local electric field variation leading to internal damage locally, and therefore significant destruction can result to the insulation over a period of time [56], [78]. Though there is no control over obstructing the formation of inherent defects in the insulating material, the magnitude of charge accumulation and rate of charge dissipation can be controlled by various means such as the addition of nanofillers and direct fluorination during the material design process [72], [79].

After the charging process, the surface potential decay characteristics can be obtained experimentally by measuring the charge remaining on the surface of the dielectric material. The experimental setup shown in Figure 1 represents the typical needle plane configuration, and it is the simplest method of acquiring the charge dissipation characteristics. Charging of the polymer surface is an essential prerequisite before obtaining the decay behaviour. The charging process of insulating materials is, in general, carried out either in contact or non-contact (corona charging) method [47], [57].
The experimental conditions shown in Figure 1 were adopted to understand the study of the surface potential variation occurring due to the charge deposited by corona activity. The corona charging process is a non-destructive, convenient and widely adopted method, which has a plethora of advantages over the contact method, which includes: a) utility to check surface trap characteristics for different voltage profiles. (eg. DC, transient and composite voltages), b) flexibility in adjusting the magnitude of the applied voltage, c) accessibility in varying the applied voltage polarity, i.e. positive and negative polarities, and d) adaptability to test under varying temperatures and different ageing conditions, i.e. ultra violet (UV), gamma irradiation, water and corona ageing etc. while maintaining the uniform atmospheric experimental conditions [80]. Since one side of the surface of the specimen is open to the surrounding ambience and the other side surface is resting on the metal electrode during the charging process, the arrangement is referred to as open circuit configuration [63].

The combination of the function generator and High voltage (HV) amplifier setup generates the desired voltage signal with the required magnitude and polarity that is ready to impinge over the surface of the specimen through the needle electrode. The customized input such as pulsed, transient, or composite voltages having varied magnitudes and polarities can be generated with the help of a function generator along with the HV power supply combination besides testing alone with the DC input. Since the discharges are initiated from the sharp protrusions, the needle electrode with a tip radius of $0.3 \mathrm{~mm}$ is utilized in the present experimental setup. Mesh grid can be used in between the needle electrode and the specimen for uniform charge distribution over the surface of the sample [1], [47]. Since the discharge is controllable, based on the magnitude and polarity of the applied voltage, the produced electric field directs the charge carriers to scatter over the specimen surface. The distance between the tip of needle electrode and specimen surface is adjusted in such a way that a) the produced electric field should spray the charges over the surface instead of spraying in the surrounding environment and $b$ ) the produced electric field is just enough for the sprayed charges to settle over the localized surface states in the specimen [81], [82]. It is observed that the applied electric field below the threshold level of the material restricts the formation of space charge in bulk. Thus, the applied field well below the threshold electric field will ensure the sprayed charges settle over the surface [83]. Figure 2 shows the laboratory experimental setup for measuring the surface potential decay measurements of insulating materials through needle plane configuration method.

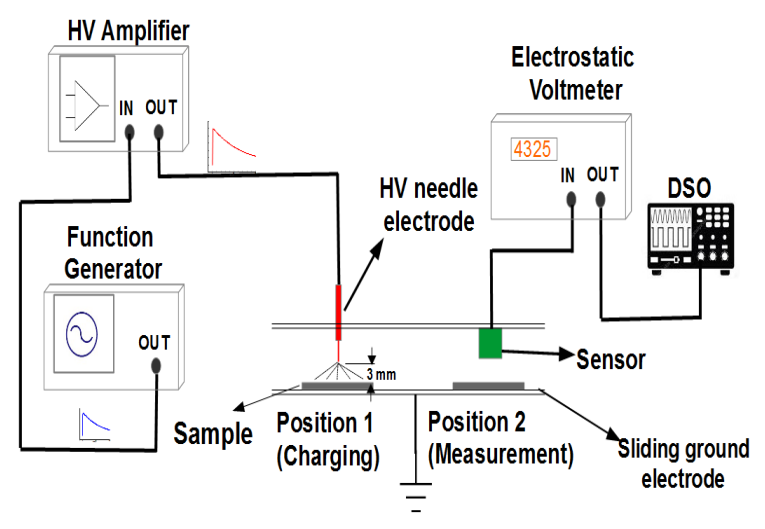

Fig. 1. Graphical experimental setup for surface potential decay studies. 


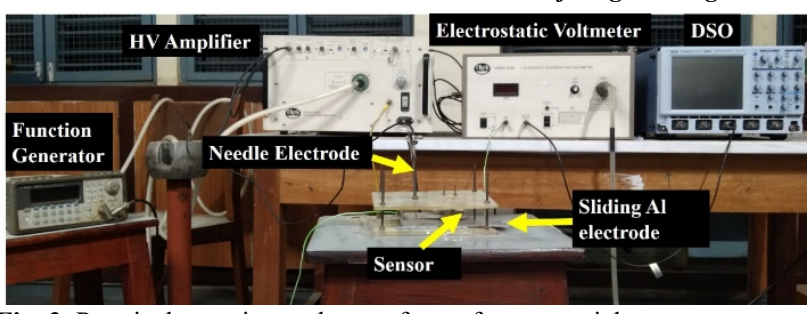

Fig. 2. Practical experimental setup for surface potential measurements.

The process by which the charges are deposited over the surface of the specimen under the corona injection from the needle electrode is illustrated in Figure 3. Collision of charges emitted from the needle electrode with the neutral atoms in the surrounding ambience results in the formation of ions (ionization) and electrons. Considering negative DC as input, the electrons and negative ions will be deposited over the surface [84]. At the same time the positive ions are also generated as a result of affinity of the molecules towards the electrons in the surrounding air. Involvement of stampedes of charges in reiterating collisions and recombination yields the charge deposition over the specimen surface. During the process, all the charges may not reach the surface of the specimen due to the loss of energy during the collisions, ionic formation and recombination. Thus, the trajectory of charge is dictated by the electric field, and the corresponding discharge, in general, possesses the same polarity as that of the high voltage electrode.

Thermal agitation due to the collision of stampedes of charges originating from the corona needle electrode forces the charges deposited earlier over the surface to transport towards the bulk of the material. Since the practical polymeric materials are the aggregate structures of amorphous and/or polycrystalline nature, the charge carrier trapping is primarily characterized by the localized states that arise from the various defects. The defective states essentially classified into two categories i.e. physical and chemical defects [75]. The physical defects include structural imperfections due to static or induced disorders at the grain boundaries, intentional (nanofillers, silane compounds and curing agents etc.) and unintentional (voids, agglomerations, moisture and dust particles etc.). Chemical defects comprise charge carrier interaction with the polar molecules in the resultant material, a dynamic disorder caused due to molecular lattice vibrations due to thermal agitation, chemical tails of macromolecular chains, and van-der-Waals bonded molecular motion etc. [47], [85].

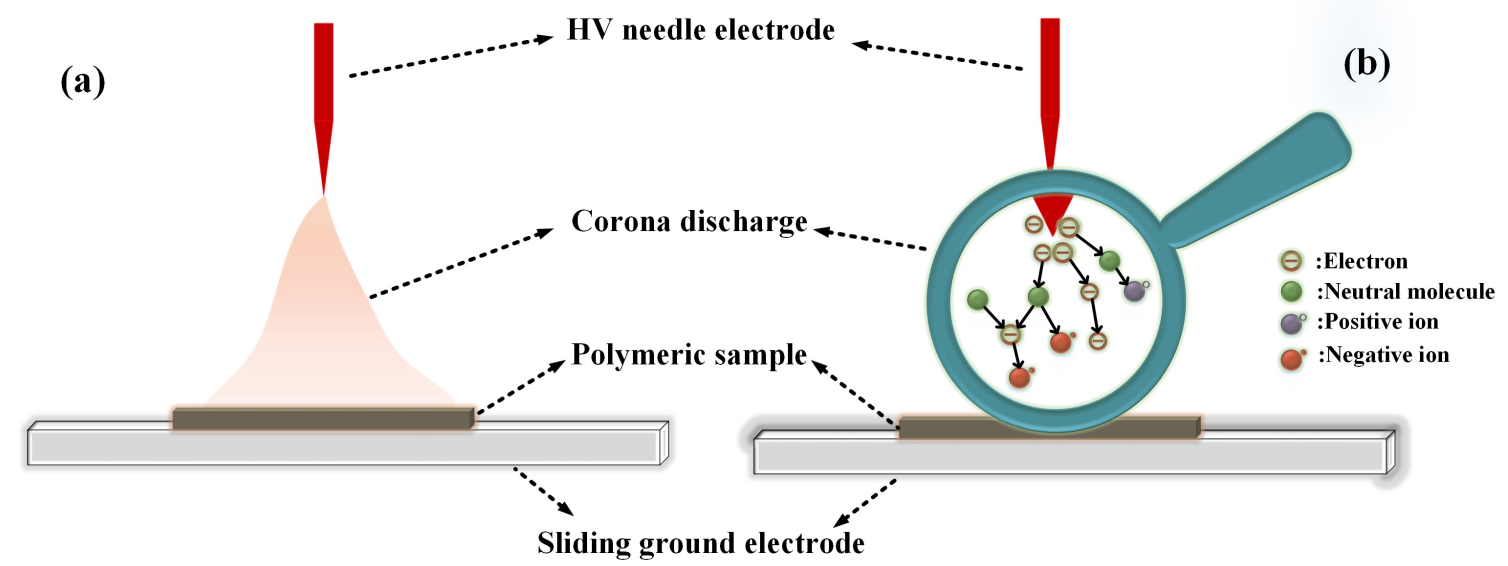

Fig. 3. Typical corona discharge (a) from the needle electrode and (b) the charge deposition process over the surface of the specimen

The process of charge carrier trapping or accumulation at the defective states defines the respective energy gap of the polymeric material [75]. The charges migrating from one side of the surface to the other side will be trapped at several defective states in the material. Upon receiving sufficient energy due to collisions from the newly coming charge carriers, the trapped charge will be released from its site and start moving towards the counter electrode, having the next higher energy site deeper in the energy gap. Thus, the charge occupying at the deeper trap sites requires higher energy to release from its localised state [86]. The process of trapping and releasing from the localized states will be continued till there is no further thrusting force either from the deposited charge carriers over the surface or from the corona electrode.

\section{Surface potential decay studies}

\subsection{Surface potential measurement}

After the charge is implanted over the surface for a specific duration of time, the potential over the surface is measured by the Kelvin probe technique by shifting the sample position from position 1 to position 2. Though other techniques such as induction probe and field mills probe are available, the
Kelvin type probe method allows the more accurate, nondestructive and non-contact type of investigating the surface charges over the polymeric surface [57], [87]. The charges over the polymeric surface induce charge on the metal electrode of the Kelvin probe which is placed above the studied surface. The probe vibrates perpendicularly to the studied surface and hence nullifying the resultant current by adjusting the voltage between the probe and electrode to zero, results in the charge over the tested surface [57]. The potential (V) over the test surface is estimated through the expression,

$V=\frac{d}{\varepsilon} \sigma$

Where $\mathrm{d}$ is the air gap between the probe and tested surface, $\varepsilon$ is the relative permittivity of the material between electrodes. $\sigma$ is the surface charge density over the material surface. Proportionally, the charge on the test surface over the scan area (A) is calculated through the expression, [88]

$$
Q=V \frac{\varepsilon A}{d}
$$


Thus, the surface charge and surface potential measured from the voltmeter are related directly to one another in the current experimental scenario.

\subsection{Mechanisms of charge decay:}

The charge being deposited and trapped near surface of the insulator, it is now free to escape from their existing states under the absence of electrical field, referred as detrapping, which involves several mechanisms such as surface conduction, bulk neutralization and gas neutralization [63], [89]. All these three phenomena are involved in the decay process. Literature reveals that one or two of the phenomena are taken in to account, and it solely depends on the specimen under test and underlying experimental conditions [63]. Uneven charge distribution of implanted charges over the specimen yields the leakage of charges along the surface termed as surface conduction. The surface conduction is strongly dependent on atmospheric conditions such as humidity, temperature and pollution levels [90], [91]. This mechanism can be ignored by maintaining the uniform experimental conditions while investigating the comparative study of decay characteristics of various polymeric materials. Gas neutralization is the process where the loosely trapped charge carriers initially detrap from their localized states by recombining with the free ions that are attracted to the polymeric surface at the gas-solid interface followed by the tightly bound charges etc. [63], [92]-[94]. Here, the ions are produced during the charge injection from the needle electrode and occupy the surface states and the charge carriers. Thus, gas neutralization is a strong function of ions present in the surrounding ambience due to the applied electric field.

On the other hand, few of the injected charges are transported to the bulk despite the control over the applied electric field due to the longer durations of charge injection and material intrinsic conductivity characteristics [19], [58]. Thus, bulk neutralization can be observed within the bulk volume of the polymeric material, where the neutralization of trapped charges is achieved through the polarization and recombination process [59]. Further, the rate of charge injection, transportation, and mobility of carriers within the bulk of respective polymeric material defines the rate of charge recombination [47]. To conclude, the deposited charges choose to decay either through the atmosphere, along the surface or across the volume based on the experimental conditions [95].

Under the absence of excitation, the trapped charges are released from their localized states through various routes such as thermal detrapping and photon assisted detrapping, tunnelling and impact ionization [47], [58]. The detrapping process associated with photons excited due to visible light in the present experimental conditions could be very slow. The available energy with the photons considering experiment at isothermal conditions is very low to stimulate the charge carriers to release from their localized states. Therefore, photon assisted detrapping can be neglected. Tunneling and impact ionization processes occur at very high electric fields. Since the applied electric field is far less than the threshold level of the material, tunneling and impact ionization processes too have the least significant effect at such a lower field in detrapping the carrier from their localized states. Thus, thermal or phonon-assisted detrapping is the possible mechanism for charge detrapping, where the energy for trapped carriers is acquired from the thermal lattice vibrations (phonons) [47].

\subsection{Trap distribution characteristics}

The characteristics of the material are well explained through the trap distribution parameters for which measurement of trap depth from the surface potential decay characteristics is a necessary requirement. Trap depth is the amount of energy required for a trapped carrier to be released from the trapped site [86]. The description of volume traps and detrapping from the demarcation energy model is first proposed by Simmons and Tam for semiconductor and insulating materials [52], [53]. Further, the model was adopted by Watson to determine the trap parameters of polystyrene material under the electron beam charging technique [54], [55]. Molinie group has studied the surface charge modelling process and demonstrated the surface demarcation energy model to characterize insulating film behaviour during the potential decay [18]. It is well known that the trapped charges having the energy above a certain limit, called the demarcation energy $\left(E_{d}\right)$, can be detrapped. Therefore, the emission of charges from the states below the demarcation level can be neglected [92]. Here $E_{d}$ defined as the border between emptied and occupied states, and its position is varied as a function of time and availability of density of trapped charges at the surface level of the respective material [54], [96]. Thus, the carriers nearing the $E_{d}$ can detrap from their localized states, resulting in detrapping emission current $\left(I_{d}\right)$. Considering the detrapping process causing the decrease in surface potential, the rate of change of potential is directly proportional to the detrapping emission current, i.e.

$I_{d}(t) \propto \frac{d V}{d t}$

Since the detrapping of charges released from the localized states is varying, the corresponding position of the $\mathrm{E}_{\mathrm{d}}$ also varies with time [53]. Furthermore, the variation in density of charges released from trap sites is directly proportional to detrapping emission current, i.e.

$$
I_{d}(t) \propto N\left(E_{d}(t)\right) \frac{d E_{d}(t)}{d t}
$$

The trap depth, which is the difference between the energy levels of conduction $\left(E_{c}\right)$ and demarcation $\left(E_{d}\right)$ states exist in the material is given by,

$$
\Delta E=E_{c}-E_{d}=k T \ln (v t)
$$

Where $\mathrm{k}$ (in $\mathrm{J} / \mathrm{K}$ ) is the Boltzmann constant, $\mathrm{T}$ (in $\mathrm{K}$ ) is the thermodynamic temperature and $v$ is the attempt to escape frequency, in order of $10^{12} \mathrm{~s}^{-1}$.

Taking the proportionalities from (3) and (4) yields

$$
\frac{d V}{d t} \propto N\left(E_{d}(t)\right) \frac{d E_{d}(t)}{d t}
$$

Differentiating equation (5) with respect to ' $t$ ' and substituting it in equation (6) gives that the density of charges at the demarcation level [56], i.e.

$N\left(E_{d}(t)\right) \propto t \frac{d V}{d t}$

Considering the trapped charges can escape from their localized states, the density of charges can be computed by [13], 


$$
N(E)=\frac{4 \varepsilon_{0} \varepsilon_{r}}{e L^{2} k T}\left|t \frac{d V(t)}{d t}\right|
$$

where $\mathrm{L}$ (in $\mathrm{m}$ ) is the thickness of the material, $\varepsilon_{\mathrm{r}}$ is the relative permittivity of the material.

\section{Case study}

In the present study, self-passivated spherical aluminium nanoparticles of $99.9 \%$ purity with an average particle size of $40 \mathrm{~nm}$ are used in the preparation of nanocomposites. Passivation is the process of formation of a thin and dense protective layer on the nanoparticle surface at the time of particle production. It provides uniform particle size distribution, besides preventing the oxidation process [97]. The presence of a passivated layer over the metallic core nanoparticles results in reduced dielectric loss over its unpassivated particles in a dielectric material [98]. Aluminium nanoparticles possess high combustion enthalpy, due to which these particles and corresponding composites find their prominence in rocket propellants, explosives, pyrotechnics, electric energy storage capacitors, embedded capacitance materials, EMI shielding applications and insulation structures. Sindhu et al. studied the influence of the addition of different sizes and concentrations of aluminium nanoparticles to the epoxy polymer matrix to vary the electric energy density for the energy storage capacitance applications [99].

Literature reveals that the controlled addition of various nanoparticles to the base polymer matrix resulted in enhanced mechanical, thermal and dielectric properties [7] [101] [98]. In specific, utilisation of passivated core-shell structured aluminium nanoparticles proved its significance in improving thermal conductivity, mechanical strength and dielectric properties of a resultant composite system. Despite the advantageous features of metallic composites below the percolation threshold in reducing dielectric loss and improved thermal conductivity, their fruitful utilization, specifically in insulation engineering, have not been explored to the core, apart from the fundamental dielectric characterization. Considerable research work is need to be carried out regarding $\mathrm{Al} /$ epoxy nanocomposites for their suitable usage as an insulating material in the electrical insulation system. Hence the present is study is marking its step towards fulfilling the gap in literature to investigate the surface potential behavior of $\mathrm{Al} /$ epoxy nanocomposites under different voltages, filler addition and different dosages of gamma irradiation.

\subsection{Surface potential measurements under DC voltage}

Inspection of the surface charge decay characteristics of epoxy nano aluminium composites viz. potential decay and trap distribution properties under the DC and transient voltage for positive and negative polarity are studied in the case study. Further, one more case study is presented to understand the charge trap characteristics due to the ageing process under the gamma-irradiated environment for nanocomposite samples. The details regarding the selection of filler concentration, material preparation, microstructural characterization and ageing parameters of the materials are reported in the earlier literature [80], [102], [103]. The experimental setup depicted [47]in Figure 1 was employed to investigate the surface potential decay characteristics of the nanocomposites for the positive and negative polarity of the applied voltage. Though the details of the experimental setup are discussed earlier, specific experimental conditions are provided in this section. All the specimens of nanocomposite materials are sliced into square-shaped with the dimensions of $40 \times 40 \mathrm{~mm}$ for the surface charge measurements. One side of the nanocomposite sample surface is exposed to air and charge is implanted from the needle electrode. Another side of specimen surface is resting on the sliding aluminium sheet. The combination of a function generator (AFG3051C) and a high voltage amplifier (Trek model 20/20 C) is used to supply the required voltage to the needle electrode. Three minutes of charging time are chosen, during which the specimen remains at position 1 . The specimen is shifted to position two, where the potential remaining on the surface of the specimen is measured upon shifting the sample to position two after the charging process under isothermal conditions. The surface potential is measured though the Kelvin probe which is connected to an electrostatic voltmeter (Trek model 341B). Subsequently, data is recorded continuously in the oscilloscope. Further, the dielectric response of the materials investigated in the earlier studies is utilized for understanding the variation in trap parameters of the respective materials [80], [103].

As discussed earlier, the charge carrier trap formation in the polymeric material is due to physical and chemical defects, and the injected carriers accumulate at the boundaries of these defective sites due to thermal agitation of charge collisions resulting from the needle electrode. The applied voltage from the needle electrode is sufficient enough for the charge carriers to be deposited over the surface, whereas the resultant field is insufficient for the injected charges to transport through the bulk of the material. Thus, the bulk neutralization and surface conduction are considered to be of the least significance for the trapped charges to release from their localized trapping sites [47]. Since the objective is to observe the surface potential variation, the present experimental electrode configuration can be used to obtain the charge trap characteristics. Therefore, the charge decay essentially occurs through the phonon-assisted detrapping, where the trapped charge is estimated to decay by recombining with the ions of opposite sign in the surrounding ambience, under the absence of applied field conditions. The respective potential measured through the electrostatic voltmeter at any instant of time is in proportion with the charge detected over the surface of the specimen through the Kelvin probe, which is positioned at $3 \mathrm{~mm}$ above the charged surface of the specimen.

The surface potential decay from the trapping sites on the surface of the different nanocomposite materials is plotted in Figure 4 for the applied DC voltage profiles under positive and negative polarities. The surface potential is decayed exponentially as a function of time for all the samples under observation regardless of the applied polarity. Thus, the decay studies are analyzed mathematically upon fitting the recorded surface potential through the exponentially decaying function [80],

$V(t)=V_{0} e^{-\frac{t}{\tau}}$

where $\mathrm{V}_{0}$ corresponds to the initial surface potential measured at the charged specimen surface after being brought under the Kelvin probe. The variable $\tau$ (in s) represents the mean lifetime of the carriers, i.e. the time taken for the potential decay curves to reach around $37 \%$ of initial potential. Furthermore, a variable $\lambda$ (in $\mathrm{s}^{-1}$ ) is referred to as decay rate constant, and it can be defined as the inverse of the mean lifetime of the carriers $(\tau)$. From the experimental results, it is seen that the potential decay is rapid during the initial period 
of time, and it becomes slower afterwards. It is known that the presence of physical and chemical defects lead to the formation of traps, and hence the characteristics of the material are now defined by the variation in trap distribution due to the addition of nanoparticles. It is also known that charges occupy the traps having different energy levels present in the material. The presence of physical and chemical defects defines the traps with different energy levels [21]. As a result, the charges trapped at loosely bounded states require less energy to detrap in comparison with the charges trapped at sites having higher energy levels. Thus, a classification is imposed on the trap sites depending on their energy levels, wherein the trap sites with lower energy are referred to as shallow traps. On the other hand, the traps that possess higher energy are referred to as deep traps. Zhou et al. have related the shallow traps, which are higher in density in the material, are caused due to physical defects, whereas the deep traps that arise in the materials are due to chemical defects [21]. Thus, the faster potential decay shown in Figure 4 during the initial period of time is due to the detrapping of charges from the shallow traps, while the subsequent slower decay is ascribed to the decay of charges from the deep trap sites [104]. A slight variation in the magnitude of initial potential is observed for a given material with respect to a change in polarity of the applied voltage. The magnitude of initial potential under -DC is higher than $+\mathrm{DC}$. The probable reason could be affinity of the material towards the negative charge transportation during corona charging, and hence detrapping is quite difficult for negative charges in comparison with that of positive charges. Further, the charge density in the negative corona is higher than that of positive corona discharge [105]-[107].
The decay time of the nanocomposites subjected to $+\mathrm{DC}$ and -DC voltage is presented in Table 1. The decay behaviour exhibited by the epoxy nanocomposites is almost similar in both the polarities. It can be observed that the decay time is decreased from the base epoxy material to the nanocomposite having the $5 \mathrm{wt} \%$ nanofiller concentration. Further addition of nanofiller above $5 \mathrm{wt} \%$ has shown the increased decay time. Variation in the decay time for the nanocomposites as a function of filler concentration is further explained better with the determination of trap characteristics.

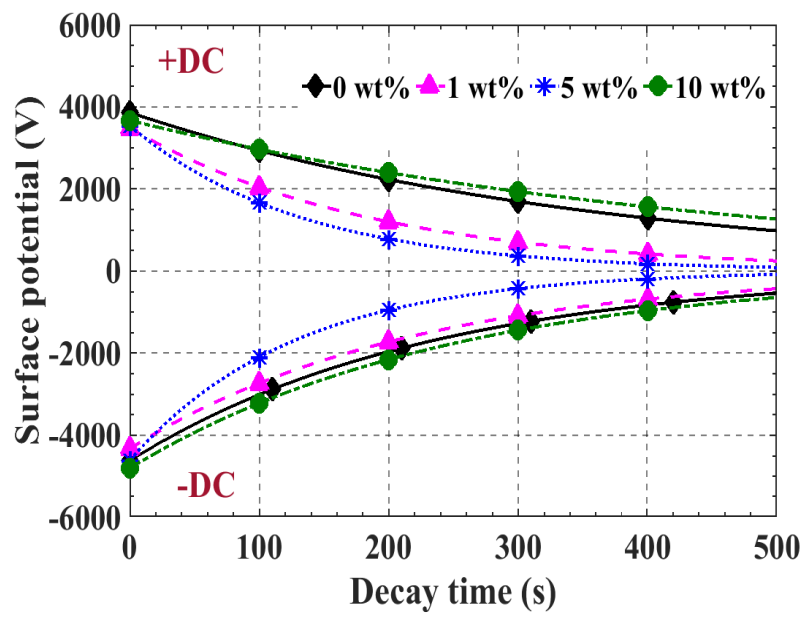

Fig. 4. Surface potential decay characteristics for $+D C$ and $-D C$ input voltage [82].

Table 1. Mean life time and trap parameters of Al/Epoxy nanocomposites under DC voltage [82].

\begin{tabular}{c|c|c|c|c}
\hline Polarity & \multicolumn{2}{|c|}{ Positive polarity } & \multicolumn{2}{c}{ Negative polarity } \\
\hline \multirow{2}{*}{ wt\% } & Mean life time (s) & Trap depth, eV & Mean life time (s) & Trap depth, eV \\
\hline 0 & 362.71 & 0.87 & 276.40 & 0.86 \\
1 & 186.85 & 0.85 & 218.15 & 0.85 \\
5 & 131.73 & 0.84 & 127.03 & 0.84 \\
10 & 468.16 & 0.87 & 250.10 & 0.86 \\
\hline
\end{tabular}

The density of charges trapped at various energy levels due to the traps that arose as a result of defects is calculated for the nanocomposites through the equation (8). Figure 5 shows the distribution of charge carrier density $(\mathrm{N}(\mathrm{E}))$ at different energy levels (E) for the Al epoxy nanocomposite materials. Here, trap depth is the energy required to liberate a charge from the trap site, i.e. lower values of trap depth indicate the minimum energy required for a charge to release from its localized state. Further, the trap center is identified as the trap depth at which maximum charge carrier density exists. A left shift in the trap center for the nanocomposites shows the enhanced charge detrapping from the localized states due to reduced trap depth. On the other hand, the right shift in the trap center shows the slower detrapping of charge. It is seen from Figure 5 that the trap center is reduced with the addition of $\mathrm{Al}$ nanoparticles to $5 \mathrm{wt} \%$ to the epoxy polymer matrix, whereas it is increased for $10 \mathrm{wt} \%$ beyond the trap depth of unfilled epoxy material. Trap centers for the nanocomposites are presented in Table 1 . It is seen that the trap distribution for epoxy nanocomposites under both the polarities is ranging from 0.7 electron Volts $(\mathrm{eV})$ to $0.9 \mathrm{eV}$.
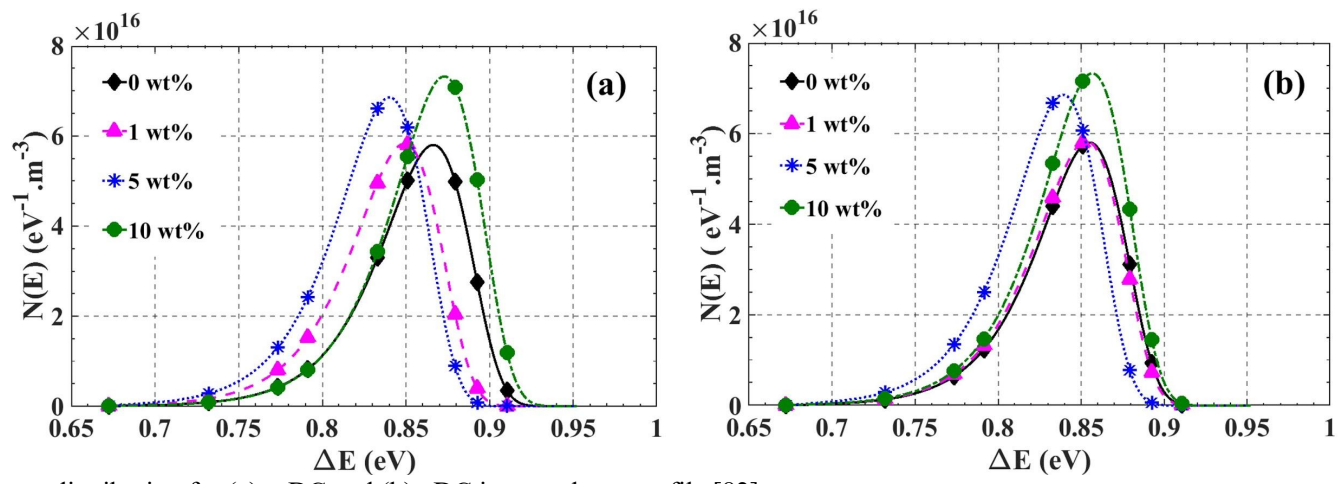

Fig. 5. Trap energy distribution for (a) + DC and (b) - DC input voltage profile [82]. 
Intuitively, the addition of nanofiller to the base polymeric material leads to the reduction of interparticle distance. Beyond a certain level of filled addition results in the aggregation of nanoparticles, and therefore, the formation of conductive channels in the nanocomposite material is inevitable. In parallel, the interfacial region formed around the nanoparticle in the polymer matrix is also a function of filler concentration. The formation of interfacial regions around the nanoparticles provides mean free path for the trapped carriers to release from their localized states. The interfacial volume is modelled as a function of filler concentration to the polymer matrix, and it is concluded that the addition of nanoparticles has shown the enhancement in the interfacial volume till certain $\mathrm{wt} \%$ of filler addition, beyond which the interfacial volume has reduced [102], [108]. Thus, enhancement in the interfacial volume due to the addition of $\mathrm{Al}$ nanoparticles till $5 \mathrm{wt} \%$ has provided the mean free path for the trapped carriers to detrap and therefore, reduction in the trap depth is observed. Whereas, the decreased interfacial volume due to agglomerations in the polymeric material resulting from the addition of nanoparticles beyond $5 \mathrm{wt} \%$ has shown the increased trap depth, in which case the escape of trapped charges is relatively difficult. The microstructural characteristics of $\mathrm{Al}$ epoxy nanocomposites are reported in earlier studies [83]. A considerable amount of energy is needed for these charge carriers trapped in a cluster of localized states to get liberated, and therefore the trap energy is increased for $10 \mathrm{wt} \%$ material. Therefore, the reduction in the trap center and reduction in the mean lifetime of the nanocomposites indicate faster detrapping characteristics. Moreover, the left shift in the trap center is intuitively thought of as the decrement in the mean lifetime of the potential decay curves.

\subsection{Surface potential measurements under transient voltages}

Considering the nanocomposite below $5 \mathrm{wt} \%$ results in better decay characteristics among the prepared specimens, the influence of variation in the transient voltage rise time is further studied for the nanocomposites. The variation in surface potential decay characteristics of epoxy nanocomposites under the transient voltage application with a front time of $0.5 \mu \mathrm{s}, 1.2 \mu \mathrm{s}, 2 \mu \mathrm{s}$ and $5 \mu \mathrm{s}$ with a constant 50 $\mu$ s tail time respectively is shown in Figure 6(a) to (d). The signal corresponding to the desired front times are generated with the help of a function generator and subsequently given to the needle electrode through the high voltage amplifier. The experimental conditions are maintained constant for all the specimens under observation. Further, the applied field conditions are sufficient for the injected charges to settle over the surface of the specimen and hence the surface conduction and bulk neutralizations are assumed to have negligible effect. The release of charge from the trapped sites is mainly through the thermal detrapping in which case the trapped charge is neutralized by recombining with the ions of opposite signs around the charged surface.
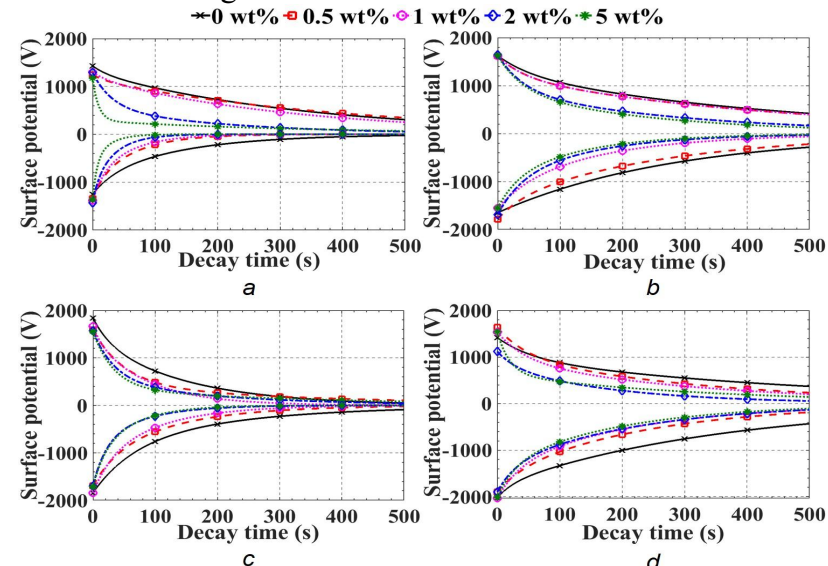

Fig. 6. Surface decay potential characteristics for transient voltage profile with (a) $0.5 \mu \mathrm{s}$, (b) $1.2 \mu \mathrm{s}$ (c) $2 \mu \mathrm{s}$ and (d) $5 \mu \mathrm{s}$ rise time [83].

Exponential decay in surface potential was observed irrespective of the applied polarity and filler concentration. Here also, the initial surface potential under the applied transient voltages is higher in a negative polarity case than in positive polarity. Similar to the DC voltage application, the inclusion of the nanofiller to the epoxy polymer matrix results in the reduced interparticle distance, giving rise to enhanced interfacial volume for the enhanced charge carrier mobility to detrap from their localized states. Hence, a decrease in a mean lifetime can be observed in all the voltage profiles and follows a similar decay pattern with the inclusion of $\mathrm{Al}$ nanofiller to the base epoxy polymer. The trap distribution characteristics shown in Figure 7 and 8 are derived from the surface potential decay curves to better picturize the variation in trap center as a function of filler concentration and rise time of the transient pulse. Variation of trap energy density for positive and negative polarities of $\mathrm{Al}$ filled epoxy nanocomposites for different transient voltage profiles is shown in Figure 7 and 8, respectively. It is seen that the trap distribution covers the range from $0.7 \mathrm{eV}$ to $0.9 \mathrm{eV}$. Table 2 shows the variation in the trap center, which represents the energy at maximum charge density, variation with respect to the addition of filler and polarity of applied voltage profiles.

Table 2. Variation in trap depth as a function of filler concentration under different transient voltage profiles [83]

\begin{tabular}{|c|c|c|c|c|c|c|c|c|}
\hline \multirow[b]{2}{*}{$\mathrm{wt} \%$} & \multicolumn{2}{|c|}{$\Delta \mathrm{E}\left(\mathrm{t}_{\mathrm{f}}=0.5 \mu \mathrm{s}\right)$} & \multicolumn{2}{|c|}{$\Delta \mathrm{E}\left(\mathrm{t}_{\mathrm{f}}=1.2 \mu \mathrm{s}\right)$} & \multicolumn{2}{|c|}{$\Delta \mathrm{E}\left(\mathrm{t}_{\mathrm{f}}=2 \mu \mathrm{s}\right)$} & \multicolumn{2}{|c|}{$\Delta \mathrm{E}\left(\mathrm{t}_{\mathrm{f}}=5 \mu \mathrm{s}\right)$} \\
\hline & $\stackrel{+}{+}$ & polarity & $\stackrel{+}{+}$ polarity & $\stackrel{-}{\text { polarity }}$ & 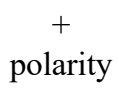 & $\begin{array}{c}- \\
\text { polarity }\end{array}$ & $\stackrel{+}{+}$ & polarity \\
\hline 0 & 0.87 & 0.83 & 0.87 & 0.86 & 0.84 & 0.83 & 0.87 & 0.87 \\
\hline 0.5 & 0.87 & 0.82 & 0.87 & 0.86 & 0.82 & 0.83 & 0.86 & 0.85 \\
\hline 1 & 0.86 & 0.81 & 0.86 & 0.84 & 0.83 & 0.82 & 0.86 & 0.85 \\
\hline 2 & 0.81 & 0.80 & 0.82 & 0.82 & 0.81 & 0.80 & 0.85 & 0.85 \\
\hline 5 & 0.77 & 0.77 & 0.82 & 0.82 & 0.81 & 0.81 & 0.79 & 0.85 \\
\hline
\end{tabular}


Chillu Naresh, Rengaswamy Jayaganthan, Ramanujam Sarathi and Michael G. Danikas/

Journal of Engineering Science and Technology Review 14 (3) (2021) 179 - 193

$* 0$ wt $\% \bullet 0.5$ wt $\% \cdot 1$ wt $\% \diamond 2$ wt $\% * 5$ wt\%

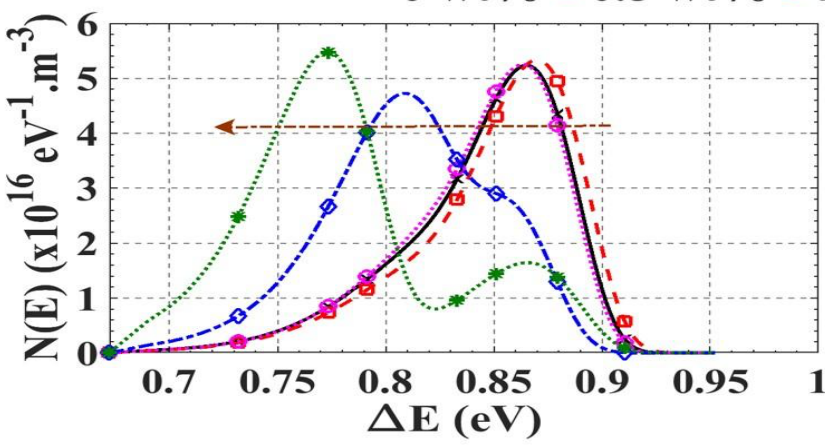

a

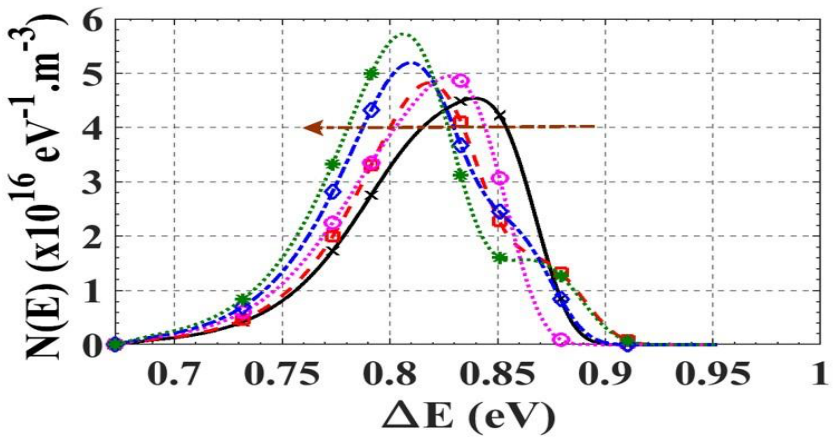

C

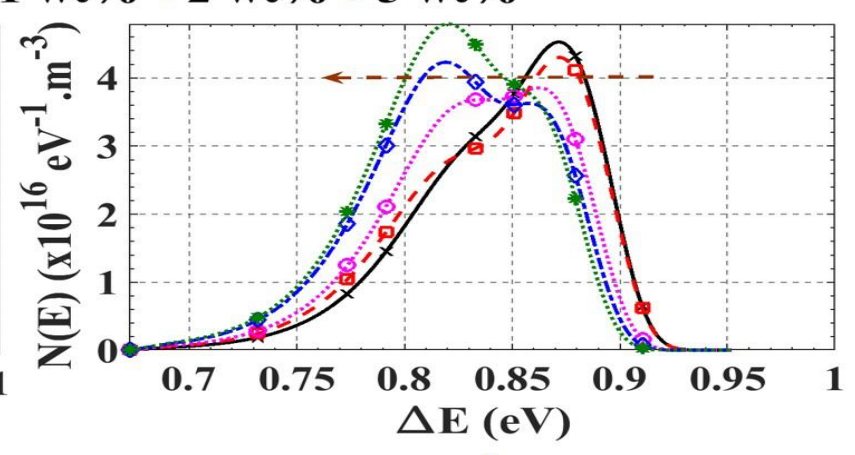

$b$

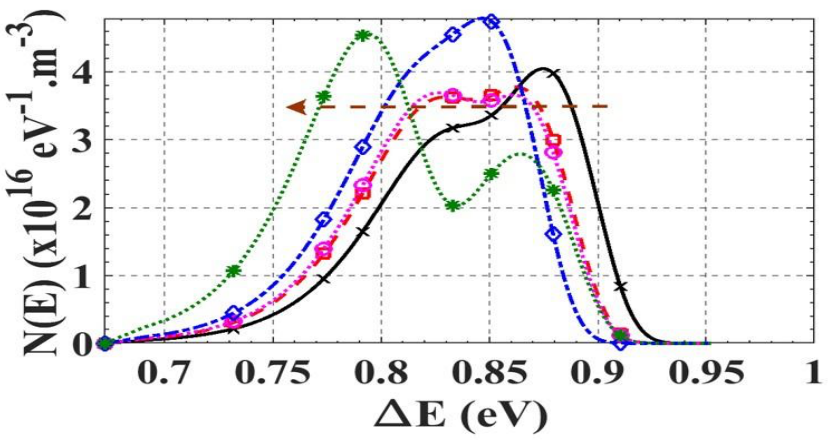

d

Fig. 7. Variation in trap energy density as a function of trap depth for a transient input with positive polarity having a rise time of (a) $0.5 \mu$ s (b) $1.2 \mu \mathrm{s}$ (c) $2 \mu \mathrm{s}$ and (d) $5 \mu \mathrm{s}[83]$.

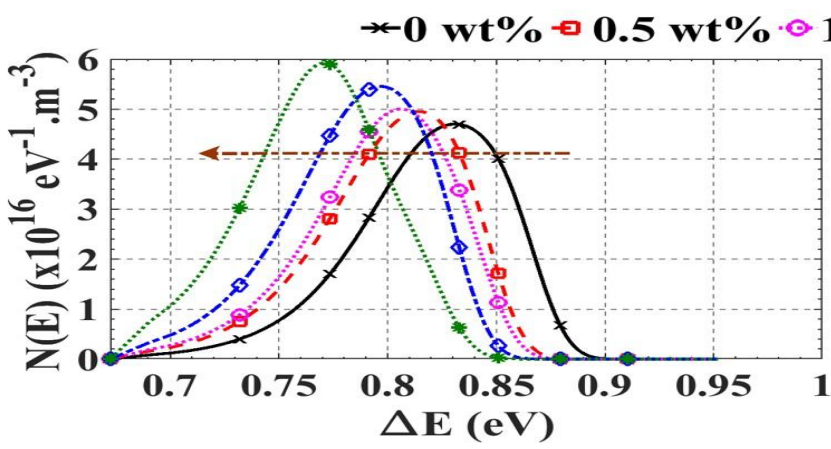

a

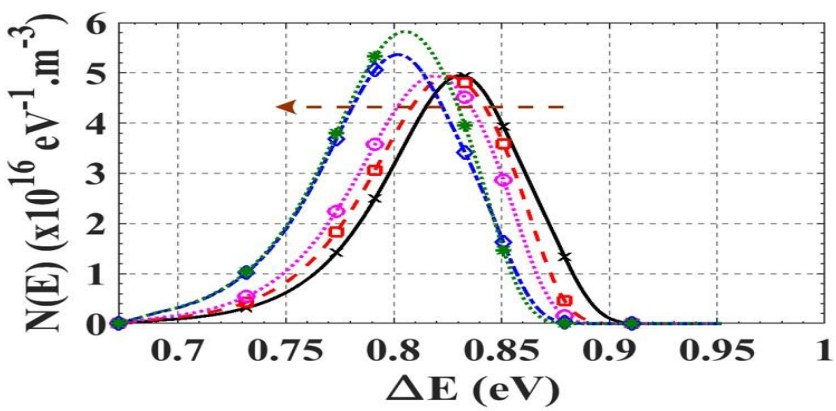

C

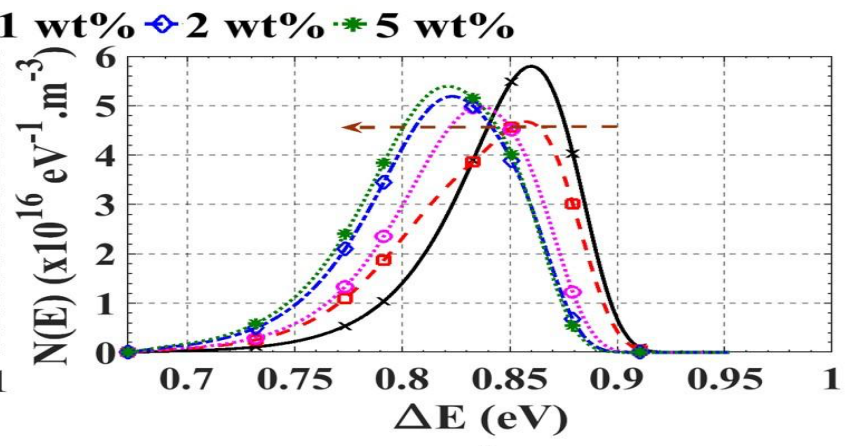

$b$

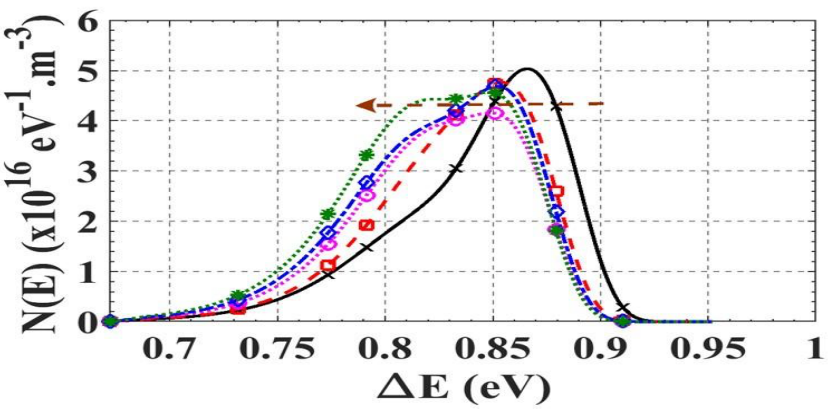

d

Fig. 8. Variation in trap energy density as a function of trap depth for a transient input with negative polarity having a rise time of (a) $0.5 \mu$ s (b) $1.2 \mu \mathrm{s}$ (c) $2 \mu \mathrm{s}$ and (d) $5 \mu \mathrm{s}[83]$.

For a given voltage profile, enhancement in interfacial volume due to the inclusion of nanofiller concentration reduces the deep traps, and it is confirmed by observing the left shift in the trap center from trap energy plots. Thus, the faster charge decay characteristics are observed for the $\mathrm{Al}$ epoxy nanocomposites under the absence of the corona injection. Further, the increased rise time of the applied voltage has induced the deep trap sites in the nanocomposite material. The reason can be explained through the core-shell structure of nanoparticles in the polymer matrix [109]. The charges are accumulated at the shallower traps present at the loosely bounded layer (the third layer) under the applied field 
conditions. The collision of charges resulted from the thermal agitation thrusting the carriers at shallower traps to migrate to deep trap states (through the second layer). Since the charge injected from the high voltage electrode is high with the increased rise time of the transient voltage, the enhancement in the charge transportation forces the charge carriers to migrate deeper and thereby raise in the deep trap energy levels are observed [83].

\subsection{Impact of gamma-irradiation on charge trap characteristics}

In this study, the surface potential characteristics of gammairradiated Al epoxy nanocomposites subjected to $10 \mathrm{kV} \mathrm{DC}$ input voltage are investigated to understand the influence of ageing parameters on the charge trap characteristics. Considering the superior trap characteristics exhibited by the $5 \mathrm{wt} \%$ Al epoxy nanocomposite sample from the earlier studies, the gamma irradiation is thus performed on the $5 \mathrm{wt} \%$ specimens along with its unfilled epoxy specimen. The prepared insulating materials were subjected to gamma irradiation through ${ }^{60} \mathrm{Co}$ source provided with the air ambience with the dosage rate of $660 \mathrm{~Gy} / \mathrm{h}$, up to the total gamma dosage of 12 kilo gray (kGy). The preceding letter ' $U$ ' and ' $\mathrm{G}$ ' in the sample notation correspond to unaged and gamma aged, respectively, and the succeeding number represents the nanofiller concentration. For example, G5 corresponds to gamma aged $5 \mathrm{wt} \%$ nano filled specimen.

The samples have shown a significant discoloration after the gamma irradiation. The variation in the physical changes due to gamma irradiation is shown in Figure 9. The virgin specimen was observed to be transparent but with the gamma irradiation, it became opaque. The cause for it is due to the radiation caused oxidation reactions to epoxy [110]. Similarly, the nanocomposite material too has shown slight change in its color, though it is slight difficult to notice due to higher opacity.

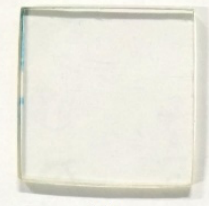

(a)

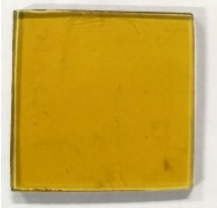

(c)

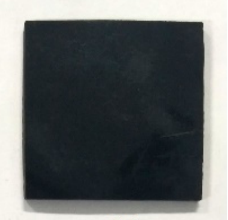

(b)

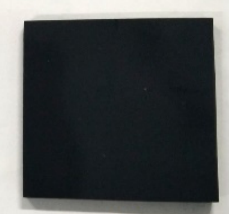

(d)
Fig. 9. Discoloration of different Al-epoxy nanocomposites due to gamma irradiation (a) U0 (b) U5 (c) G0 and (d) G5.

The surface potential characteristics of gamma-irradiated nanocomposite materials are shown in Figure 10. It is observed that the potential decays exponentially irrespective of the ageing, material and applied polarity of the voltage profile. The initial potential and decay rate variation of gamma-irradiated al epoxy nanocomposites are shown in Figure $11 \mathrm{a}$ and $\mathrm{b}$ for the applied $+\mathrm{DC}$ and $-\mathrm{DC}$ voltage. The faster charge decay characteristics of U5 is observed in comparison with the unaged epoxy polymer (U0). Similarly, G5 is showing faster decay characteristics G0. Whereas the unaged nanocomposites are exhibiting faster charge decay properties over their aged counterparts.

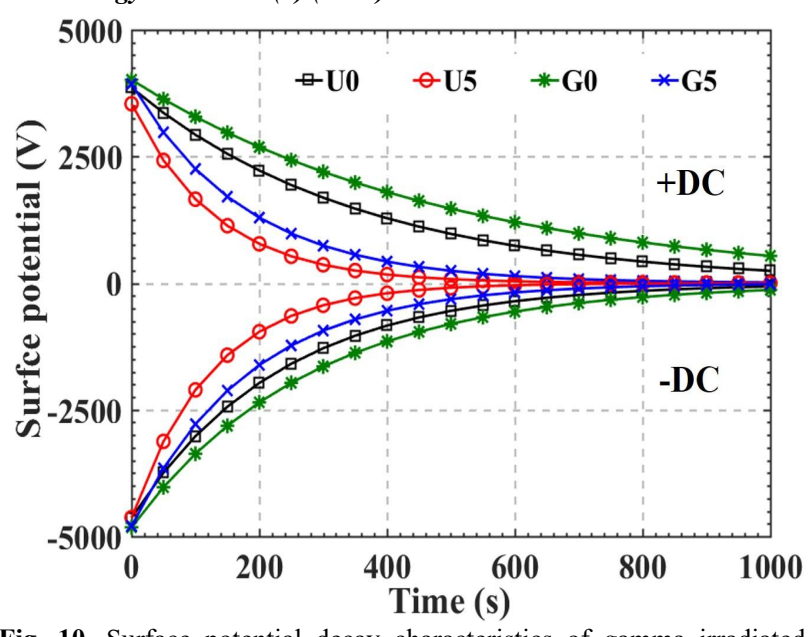

Fig. 10. Surface potential decay characteristics of gamma irradiated $\mathrm{Al} /$ epoxy nanocomposites under $+\mathrm{DC}$ and $-\mathrm{DC}$ voltage profile [103].

The trap distribution properties of gamma-irradiated $\mathrm{Al} /$ epoxy nanocomposites are represented in Figure 12a and $\mathrm{b}$, respectively. Despite the applied polarity, it is observed that the trap energy distribution covers the range from $0.7 \mathrm{eV}$ to $0.9 \mathrm{eV}$. The variation in trap depth for the materials based on their filler inclusion and gamma dosage rate is shown in Table 3. It is observed that the U5 has a lower trap center compared to the U0. Similarly, specimen G5 has a lower trap depth in comparison with specimen G0. A higher trap center is observed for aged composites over their unaged counterparts. The enhancement in the interphase area eases the charge carrier movement freely under the absence of corona charge injection. Enhancement in the carrier mobility due to available free interface volume allows the detrapping process more easily for the sample U5 compared with U0 [102], [108]. Hence, the sample U5 exhibits the lowest trap depth and lower mean lifetime. Chain scission and molecular chain breakage caused due to gamma dosage at the surface states, allowing the charge carriers to trap at the verge of broken bonds.

The degradative reaction of functional group in the nanocomposite samples is dominant due to gamma irradiation [111]. The impact of gamma irradiation induces functional group degradation and therefore, new structural defects can be formed, thereby increasing the number of localized traps [13], [112]. Thus, the charge accumulation in the case of gamma-irradiated samples is higher than that of unaged specimens. As additional trap sites are formed due to the gamma irradiation, the detrapping of carriers is therefore difficult from the deep trap sites. Thus, it is evident that the rightward shift in the trap center can be seen in the trap energy distribution plot, as shown in Figure 12 for the gamma aged samples.

Even though the gamma irradiation increases the trap depth for unaged epoxy specimens, the same is not followed for the G5 specimen. Improved interfacial volume due to nanofiller inclusion at $5 \mathrm{wt} \%$ alleviates the increased trap depth. To conclude, the addition of nanofiller to the polymer matrix can tailor the trap distribution characteristics both in unaged and gamma aged environments. Also, it is further observed from Table 3 that the trap depth and mean lifetime followed the direct correlation. The sample with a lower trap center is exhibiting a lower mean lifetime and vice versa. 

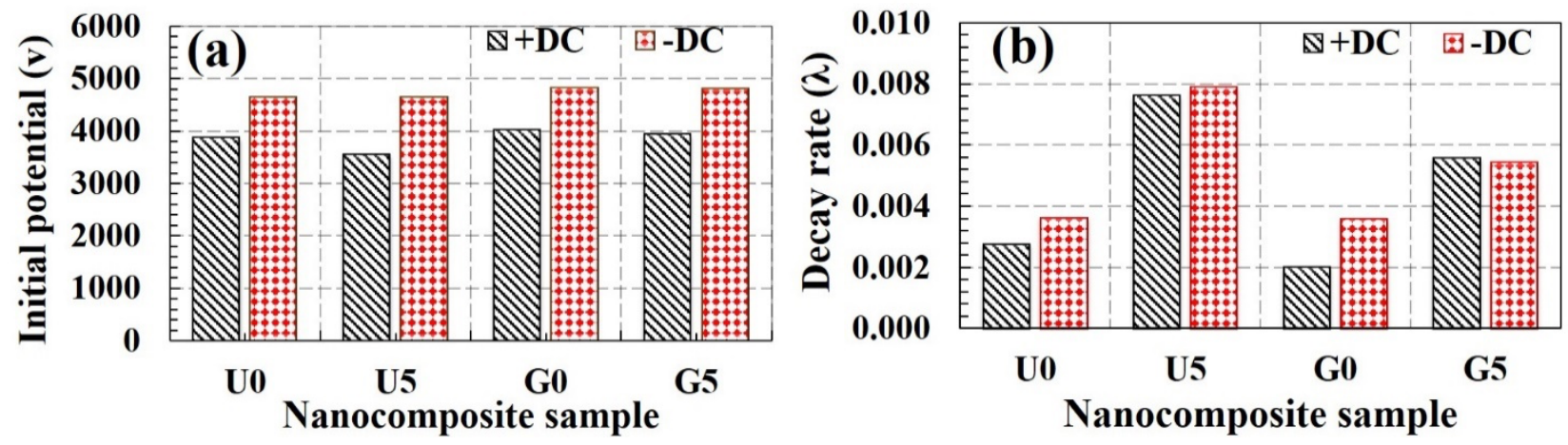

Fig. 11. Variation in (a) initial surface potential and (b) decay rate constant for the normal and gamma-irradiated nanocomposite samples [103].
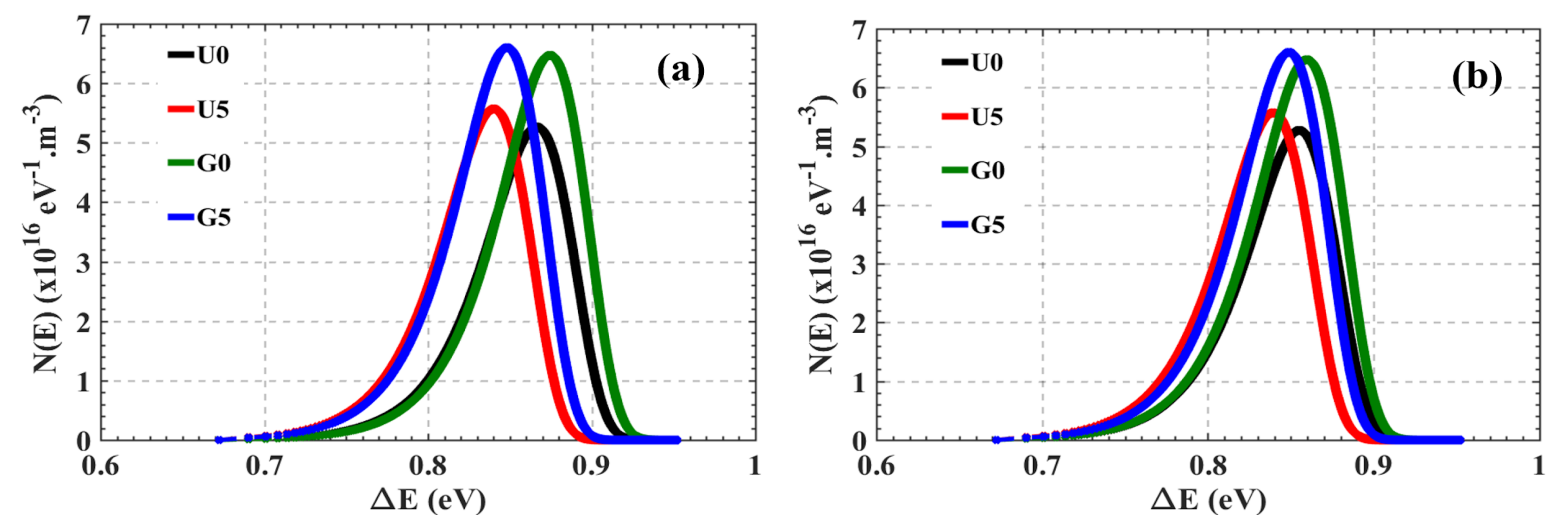

Fig. 12. Trap energy distribution of gamma irradiated nanocomposites for (a) + DC and (b) -DC input [103].

Table 3. Mean lifetime and trap centres of Al/epoxy nanocomposite samples [103].

\begin{tabular}{|c|c|c|c|c|}
\hline Polarity & \multicolumn{2}{|c|}{ Positive polarity } & \multicolumn{2}{|c|}{ Negative polarity } \\
\hline Sample & Mean lifetime (s) & Trap depth, eV & Mean lifetime (s) & Trap depth, eV \\
\hline U0 & 362.71 & 0.87 & 276.40 & 0.86 \\
\hline U5 & 131.73 & 0.84 & 127.03 & 0.84 \\
\hline G0 & 497.51 & 0.87 & 279.02 & 0.86 \\
\hline G5 & 179.79 & 0.85 & 183.82 & 0.85 \\
\hline
\end{tabular}

\section{Conclusions}

The main goal of the present work is to bring the consolidated review on methods of surface potential measurements performed on various polymeric materials, mechanisms of charge decay and trap distribution associated with the respective materials. The variation in the surface potential as well as the respective trap distribution due to the $\mathrm{Al}$ nanofiller addition, under different voltage profiles and different irradiated environments are elucidated. The important reviewed topics and the main contributions of the present research on polymeric materials in the viewpoint of insulating applications based on the studies are the following:

- The experimental setup of surface potential measurements is explained through the simple and noncontact type of needle plane electrode setup. Surface potential measurement is discussed briefly through the vibrating reed principle adopted in the Kelvin probe in determining the surface charge.
- The various charge neutralization mechanisms such as gas, bulk and surface conduction processes are reviewed and hence it is highlighted that the dominance of one of the mechanisms during the surface potential decay depends on the type of specimen under test and the underlying experimental conditions. Further, the phononassisted detrapping is seemed to be the possible charge release method from the surface states upon removal of the excitation source.

- $\quad$ The charge parameters such as mean lifetime, trap center, initial potential and decay rate are discussed from the trap distribution analysis to characterize the surface charge decay behaviour of polymeric materials.

- The three case studies are discussed in order to examine the influence of nanoparticle addition, variation in voltage profile and gamma ageing effect on trap distribution characteristics of $\mathrm{Al}$ filled epoxy nanocomposite materials.

- Controlled addition of nanoparticles to certain wt $\%$ has enhanced the detrapping of carriers from trap sites due to increased interfacial volume. 
- Increased rise time in the input transient voltage profile has resulted in the increased trap energy states.

- Despite the gamma irradiation induce an additional traps in accumulating more charges, the effect is nullified by the controlled addition of nanoparticles to the epoxy base matrix.
- $\quad$ Thus, the addition of nanoparticles to the polymer matrix plays an important role in tailoring the trap characteristics of resulting nanocomposite materials.

This is an Open Access article distributed under the terms of the Creative Commons Attribution License.

\section{References}

1. J. A. Giacometti and O. N. Oliveira, "Corona charging of polymers," IEEE Transactions on Electrical Insulation, vol. 27, no. 5, pp. 924 943, 1992.

2. J. Mort, "Xerography: A Study in Innovation and Economic Competitiveness," Physics Today, vol. 47, no. 4, pp. 32-38, Apr. 1994.

3. D. M. Burland and L. B. Schein, "Physics of Electrophotography," Physics Today, vol. 39, no. 5, pp. 46-53, May 1986.

4. R. H. Young, "Kinetics of xerographic discharge by surface charge injection," Journal of Applied Physics, vol. 72, no. 7, pp. 2993-3004, Oct. 1992.

5. K. Yasumoto, A. Zukeran, Y. Takagi, Y. Ehara, T. Takahashi, and T. Yamamoto, "Effect of electrode thickness for reducing ozone generation in electrostatic precipitator," Electronics and Communications in Japan, vol. 93, no. 7, pp. 24-31, Jun. 2010.

6. A. S. Viner, P. A. Lawless, D. S. Ensor, and L. E. Sparks, "Ozone generation in DC-energized electrostatic precipitators," in Conference Record - IAS Annual Meeting (IEEE Industry Applications Society), 1989, no. pt 2, pp. 2167-2174.

7. A. Gerlach, M. Liebler, G. M. Sessler, H. von Seggern, B. Scheufele, and E. Hirth, "Comparative analysis of isothermal decay of the surface potential of fluoroethylenepropylene electrets and of the sensitivity of electret microphones at elevated temperature," AIP Advances, vol. 10, no. 9, p. 095313, Sep. 2020.

8. G. M. Sessler and J. E. West, "Foil-Electret Microphones," The Journal of the Acoustical Society of America, vol. 40, no. 6, pp. 1433-1440, Dec. 1966.

9. G. M. Sessler, "Charge storage in dielectrics," IEEE Transactions on Electrical Insulation, vol. 24, no. 3, pp. 395-402, Jun. 1989.

10. K. D. Weltmann, E. Kindel, T. von Woedtke, M. Hähnel, M. Stieber, and R. Brandenburg, "Atmospheric-pressure plasma sources: Prospective tools for plasma medicine," Pure and Applied Chemistry, vol. 82, no. 6, pp. 1223-1237, Apr. 2010.

11. M. Laroussi, "Low-Temperature Plasmas for Medicine?," IEEE Transactions on Plasma Science, vol. 37, no. 6, pp. 714-725, Jun. 2009.

12. C.-F. Liaw and I.-C. Chen, "Poling of multiple PVDF films by moving corona discharge," Ferroelectrics, vol. 99, no. 1, pp. 127132, Nov. 1989.

13. B. X. Du, X. X. Kong, Y. Q. Xing, and J. Li, "Trap distribution of electron beam irradiated epoxy resin under repetitive pulse voltage," IEEE Transactions on Dielectrics and Electrical Insulation, vol. 24, no. 6, pp. 3869-3877, Dec. 2017.

14. J. Naveen, R. Sarathi, and T. Imai, "Understanding the impact of gamma irradiation of epoxy titania nanocomposites on surface and bulk charge characteristics," Nano Express, vol. 1, no. 1, p. 010061 , Jun. 2020.

15. T. Kurihara, T. Takahashi, H. Homma, and T. Okamoto, "Oxidation of cross-linked polyethylene due to radiation-thermal deterioration," IEEE Transactions on Dielectrics and Electrical Insulation, vol. 18, no. 3, pp. 878-887, Jun. 2011.

16. T. J. Gallagher and A. J. Pearmain, High Voltage: Measurement, Testing and Design. Wiley, 1983.

17. M. Ieda, G. Sawa, and U. Shinohara, "A Decay Process of Surface Electric Charges across Poyethylene Film," Japanese Journal of Applied Physics, vol. 6, no. 6, pp. 793-794, Jun. 1967.

18. P. Llovera and P. Molinie, "New methodology for surface potential decay measurements: application to study charge injection dynamics on polypropylene films," IEEE Transactions on Dielectrics and Electrical Insulation, vol. 11, no. 6, pp. 1049-1056, Dec. 2004.

19. P. Molinie, "A Review of Mechanisms and Models Accounting for Surface Potential Decay," IEEE Transactions on Plasma Science, vol. 40, no. 2, pp. 167-176, Feb. 2012.

20. L. A. Dissado, G. Mazzanti, and G. C. Montanari, "The role of trapped space charges in the electrical aging of insulating materials," IEEE Transactions on Dielectrics and Electrical Insulation, vol. 4, no. 5, pp. 496-506, 1997.

21. G. Chen and $\mathrm{Z} . \mathrm{Xu}$, "Charge trapping and detrapping in polymeric materials," Journal of Applied Physics, vol. 106, no. 12, p. 123707 , Dec. 2009.

22. T. Kikuchi, S. Nishimura, M. Nagao, K. Izumi, Y. Kubota, and M. Sakata, "Survey on the use of non-ceramic composite insulators," IEEE Transactions on Dielectrics and Electrical Insulation, vol. 6, no. 5, pp. 548-556, 1999.

23. R. Hackam, "Outdoor HV composite polymeric insulators," IEEE Transactions on Dielectrics and Electrical Insulation, vol. 6, no. 5, pp. 557-585, Dec. 1999.

24. E. A. Cherney and R. S. Gorur, "RTV silicone rubber coatings for outdoor insulators," IEEE Transactions on Dielectrics and Electrical Insulation, vol. 6, no. 5, pp. 605-611, 1999.

25. M. Farzaneh and W. A. Chisholm, Insulators for Icing and Polluted Environments. Hoboken, NJ, USA: John Wiley \& Sons, Inc., 2009.

26. S. M. Gubanski, A. Dernfalk, J. Andersson, and H. Hillborg, "Diagnostic Methods for Outdoor Polymeric Insulators," IEEE Transactions on Dielectrics and Electrical Insulation, vol. 14, no. 5, pp. 1065-1080, Oct. 2007.

27. J.-W. Zha, Z.-M. Dang, W.-K. Li, Y.-H. Zhu, and G. Chen, "Effect of micro-Si 3 N 4 -nano-Al 2 O 3 co-filled particles on thermal conductivity, dielectric and mechanical properties of silicone rubber composites," IEEE Transactions on Dielectrics and Electrical Insulation, vol. 21, no. 4, pp. 1989-1996, Aug. 2014.

28. D. A. Swift, C. Spellman, and A. Haddad, "Hydrophobicity transfer from silicone rubber to adhering pollutants and its effect on insulator performance," IEEE Transactions on Dielectrics and Electrical Insulation, vol. 13, no. 4, pp. 820-829, Aug. 2006.

29. A. El-Hag, L. H. Meyer, and A. Naderian, "Experience with salt-fog and inclined-plane tests for aging polymeric insulators and materials," IEEE Electrical Insulation Magazine, vol. 26, no. 2, pp. 42-50, Mar. 2010.

30. B. Venkatesulu and M. Thomas, "Erosion resistance of aluminafilled silicone rubber nanocomposites," IEEE Transactions on Dielectrics and Electrical Insulation, vol. 17, no. 2, pp. 615-624, Apr. 2010.

31. I. J. S. Lopes, S. H. Jayaram, and E. A. Cherney, "A study of partial discharges from water droplets on a silicone rubber insulating surface," IEEE Transactions on Dielectrics and Electrical Insulation, vol. 8, no. 2, pp. 262-268, Apr. 2001.

32. Y. Zhu, "Influence of corona discharge on hydrophobicity of silicone rubber used for outdoor insulation," Polymer Testing, vol. 74, pp. 14-20, Apr. 2019.

33. I. Ramirez, S. Jayaram, E. Cherney, M. Gauthier, and L. Simon, "Erosion resistance and mechanical properties of silicone nanocomposite insulation," IEEE Transactions on Dielectrics and Electrical Insulation, vol. 16, no. 1, pp. 52-59, Feb. 2009.

34. F. Obenaus, "Fremdschichtueberschlag und Kriechweglaenge," Elektrotechnik, vol. 4, pp. 135-137, 1958.

35. D. Kind and H. Kärner, High-voltage insulation technology, vol. 621. Springer, 1985.

36. T. Sathishkumar, S. Satheeshkumar, and J. Naveen, "Glass fiberreinforced polymer composites - a review," Journal of Reinforced Plastics and Composites, vol. 33, no. 13, pp. 1258-1275, Jul. 2014.

37. M. Altin Karataş and H. Gökkaya, "A review on machinability of carbon fiber reinforced polymer (CFRP) and glass fiber reinforced polymer (GFRP) composite materials," Defence Technology, vol. 14, no. 4, pp. 318-326, Aug. 2018.

38. A. T. DiBenedetto, "Tailoring of interfaces in glass fiber reinforced polymer composites: a review," Materials Science and Engineering: $A$, vol. 302, no. 1, pp. 74-82, Apr. 2001. 
39. T. Paulmier, B. Dirassen, D. Payan, and M. Van Eesbeek, "Material Charging in Space Environment: Experimental Test Simulation and Induced Conductive Mechanisms," IEEE Transactions on Dielectrics and Electrical Insulation, vol. 16, no. 3, pp. 682-688, Jun. 2009.

40. C. Naresh, A. Jeshurun, R. Jayaganthan, R. Velmurugan, and R. Sarathi, "Investigation on dielectric and mechanical properties of epoxy reinforced with glass fiber and nano-silica composites," Materials Research Express, vol. 6, no. 11, p. 115082, Oct. 2019. Y. Xing, Y. Shen, X. Song, Y. Yu, and M. Xiao, "Effects of Electron Beam Irradiation on Insulation Characteristics of Epoxy/AIN Nanocomposites," IEEE Transactions on Applied Superconductivity, vol. 29, no. 2, pp. 1-4, 2019.

41. T. Tanaka and T. Imai, Advanced Nanodielectrics, 1st ed. Jenny Stanford Publishing, 2017.

42. G. Mazzanti, E. Kandia, A. Morandi, M. Fabbri, and P. L. Ribani, "Electrical insulation for high temperature superconducting fault current limiters," in 2013 IEEE International Conference on Solid Dielectrics (ICSD), 2013, pp. 337-340.

43. C. Gandioli, M. Alvarez-Herault, P. Tixador, N. Hadjsaid, and D. R. Medina, "Innovative Distribution Networks Planning Integrating Superconducting Fault Current Limiters," IEEE Transactions on Applied Superconductivity, vol. 23, no. 3, pp. 5603904-5603904, Jun. 2013.

44. T. Tanaka, G. C. Montanari, and R. Mulhaupt, "Polymer nanocomposites as dielectrics and electrical insulation-perspectives for processing technologies, material characterization and future applications," IEEE Transactions on Dielectrics and Electrical Insulation, vol. 11, no. 5, pp. 763-784, 2004.

45. Y. J. Lee et al., "High Voltage Dielectric Characteristics of Epoxy Nano-Composites in Liquid Nitrogen for Superconducting Equipment," IEEE Transactions on Applied Superconductivity, vol. 21, no. 3, pp. 1426-1429, Jun. 2011.

46. J. Li, F. Zhou, D. Min, S. Li, and R. Xia, "The energy distribution of trapped charges in polymers based on isothermal surface potential decay model," IEEE Transactions on Dielectrics and Electrical Insulation, vol. 22, no. 3, pp. 1723-1732, Jun. 2015.

47. T. Imai et al., "Improving epoxy-based insulating materials with nano-fillers toward practical application," in Conference Record of IEEE International Symposium on Electrical Insulation, 2008.

48. T. Tanaka, "Dielectric nanocomposites with insulating properties," IEEE Transactions on Dielectrics and Electrical Insulation, vol. 12, no. 5, pp. 914-928, Oct. 2005.

49. S. Singha and M. Thomas, "Dielectric properties of epoxy nanocomposites," IEEE Transactions on Dielectrics and Electrical Insulation, vol. 15, no. 1, pp. 12-23, 2008.

50. J. Li, H. C. Liang, B. X. Du, and Z. H. Wang, "Surface functional graded spacer for compact HVDC gaseous insulated system," IEEE Transactions on Dielectrics and Electrical Insulation, 2019.

51. J. G. Simmons and M. C. Tam, "Theory of Isothermal Currents and the Direct Determination of Trap Parameters in Semiconductors and Insulators Containing Arbitrary Trap Distributions," Physical Review B, vol. 7, no. 8, pp. 3706-3713, Apr. 1973.

52. J. G. Simmons, G. W. Taylor, and M. C. Tam, "Thermally Stimulated Currents in Semiconductors and Insulators Having Arbitrary Trap Distributions," Physical Review B, vol. 7, no. 8, pp. 3714-3719, Apr. 1973.

53. P. K. Watson, "The transport and trapping of electrons in polymers," IEEE Transactions on Dielectrics and Electrical Insulation, vol. 2, no. 5, pp. 915-924, 1995.

54. P. K. Watson, "The energy distribution of localized states in polystyrene, based on isothermal discharge measurements," Journal of Physics D: Applied Physics, vol. 23, no. 12, pp. 1479-1484, Dec. 1990.

55. P. Molinie, "Measuring and modeling transient insulator response to charging: the contribution of surface potential studies," IEEE Transactions on Dielectrics and Electrical Insulation, vol. 12, no. 5 , pp. 939-950, Oct. 2005.

56. P. Llovera, P. Molinié, A. Soria, and A. Quijano, "Measurements of electrostatic potentials and electric fields in some industrial applications: Basic principles," Journal of Electrostatics, vol. 67, no. 2-3, pp. 457-461, May 2009.

57. P. Molinie, M. Goldman, and J. Gatellet, "Surface potential decay on corona-charged epoxy samples due to polarization processes," Journal of Physics D: Applied Physics, vol. 28, no. 8, pp. 1601-1610, Aug. 1995.

58. P. Molinie, "Surface potential measurements: Implementation and interpretation," in Eighth International Conference on Dielectric Materials, Measurements and Applications, 2000, vol. 2000, no.

\section{3, pp. 253-258.}

59. S. Kumara, Y. Serdyuk, and S. Gubanski, "Simulation of surface charge effect on impulse flashover characteristics of outdoor polymeric insulators," IEEE Transactions on Dielectrics and Electrical Insulation, vol. 17, no. 6, pp. 1754-1763, Dec. 2010.

60. S. Alam, Y. Serdyuk, and S. Gubanski, "Potential decay on silicone rubber surfaces affected by bulk and surface conductivities," IEEE Transactions on Dielectrics and Electrical Insulation, vol. 22, no. 2, pp. 970-978, Apr. 2015.

61. C. Yuan, C. Xie, L. Li, X. Xu, and S. M. Gubanski, "Surface potential decay on material samples taken from in-service aged HVDC silicone rubber composite insulators," IEEE Transactions on Dielectrics and Electrical Insulation, vol. 24, no. 1, pp. 592-600, Feb. 2017.

62. S. Kumara, Y. Serdyuk, and S. Gubanski, "Surface charge decay on polymeric materials under different neutralization modes in air," IEEE Transactions on Dielectrics and Electrical Insulation, vol. 18, no. 5, pp. 1779-1788, Oct. 2011.

63. A. Mohamad, G. Chen, Y. Zhang, and Z. An, "Moisture effect on surface fluorinated epoxy resin for high-voltage DC applications," IEEE Transactions on Dielectrics and Electrical Insulation, vol. 23, no. 2, pp. 1148-1155, Apr. 2016.

64. B. Du and M. Xiao, "Influence of surface charge on DC flashover characteristics of epoxy/BN nanocomposites," IEEE Transactions on Dielectrics and Electrical Insulation, 2014.

65. B. X. Du, H. C. Liang, J. Li, and C. Zhang, "Temperature dependent surface potential decay and flashover characteristics of epoxy/SiC composites," IEEE Transactions on Dielectrics and Electrical Insulation, vol. 25, no. 2, pp. 631-638, Apr. 2018.

66. B. X. Du, M. Y. Wang, J. Li, and Y. Q. Xing, "Temperature dependent surface charge and discharge behavior of epoxy/AIN nanocomposites," IEEE Transactions on Dielectrics and Electrical Insulation, vol. 25, no. 4, pp. 1300-1307, Aug. 2018.

67. B. X. Du, Z. Y. Ran, J. Li, H. C. Liang, and H. Yao, "Fluorinated epoxy insulator with interfacial conductivity graded material for HVDC gaseous insulated pipeline," IEEE Transactions on Dielectrics and Electrical Insulation, vol. 27, no. 4, pp. 1305-1312, Aug. 2020.

68. B. X. Du and Y. Gao, "Gamma-ray irradiation inhibiting surface charge accumulation on polyethylene," in IEEE Transactions on Dielectrics and Electrical Insulation, 2009, vol. 16, no. 3, pp. 876881.

69. Z. L. Li, B. X. Du, Z. R. Yang, and C. L. Han, "Temperature dependent trap level characteristics of graphene/LDPE nanocomposites," IEEE Transactions on Dielectrics and Electrical Insulation, vol. 25, no. 1, pp. 137-144, Feb. 2018.

70. B. X. Du, J. Li, and W. Du, "Surface charge accumulation and decay on directfluorinated polyimide/A12O3 nanocomposites," IEEE Transactions on Dielectrics and Electrical Insulation, vol. 20, no. 5, pp. 1764-1771, 2013.

71. B. X. Du, Z. Li, Y. Gao, and X. X. Cheng, "Surface charge accumulate and decay of direct-fluorinated RTV silicone rubber," in 2013 IEEE International Conference on Solid Dielectrics (ICSD), 2013, pp. 222-225.

72. B. X. Du, X. L. Li, and J. P. Jiang, "Surface charge accumulation and decay on direct-fluorinated oil-impregnated paper," IEEE Transactions on Dielectrics and Electrical Insulation, vol. 23, no. 5, pp. 3094-3101, Oct. 2016.

73. B. X. Du, J. Li, Q. Du, and M. L. Fu, "Surface charge and flashover voltage of EVA/CB nanocomposite under mechanical stresses," IEEE Transactions on Dielectrics and Electrical Insulation, vol. 23, no. 6, pp. 3734-3741, Dec. 2016.

74. T. C. Zhou, G. Chen, R. Liao, and Z. Xu, "Charge trapping and detrapping in polymeric materials: Trapping parameters," Journal of Applied Physics, vol. 110, no. 4, p. 043724, Aug. 2011.

75. D. Fabiani et al., "Polymeric HVDC Cable Design and Space Charge Accumulation. Part 1: Insulation/Semicon Interface," IEEE Electrical Insulation Magazine, vol. 23, no. 6, pp. 11-19, Nov. 2007.

76. M. Fu, L. Dissado, G. Chen, and J. Fothergill, "Space charge formation and its modified electric field under applied voltage reversal and temperature gradient in XLPE cable," IEEE Transactions on Dielectrics and Electrical Insulation, vol. 15, no. 3, pp. 851-860, Jun. 2008.

77. Y. Gao, J. Wang, F. Liu, and B. Du, "Surface Potential Decay of Negative Corona Charged Epoxy/Al 2 O 3 Nanocomposites Degraded by 7.5-MeV Electron Beam," IEEE Transactions on Plasma Science, vol. 46, no. 7, pp. 2721-2729, Jul. 2018.

B. Zhang, G. Zhang, Q. Wang, C. Li, J. He, and Z. An, "Suppression of surface charge accumulation on Al 2 O 3 -filled 
epoxy resin insulator under dc voltage by direct fluorination," AIP Advances, vol. 5, no. 12, p. 127207, Dec. 2015.

79. C. Naresh, R. Jayaganthan, M. G. Danikas, T. Tanaka, and R. Sarathi, "Understanding the dielectric and mechanical properties of self-passivated Al-epoxy nanocomposites," in IET Science, Measurement \& Technology, 2019, vol. 13, no. 9, pp. 1336-1344.

80. S. Kumara, Bin Ma, Y. V. Serdyuk, and S. M. Gubanski, "Surface charge decay on HTV silicone rubber: effect of material treatment by corona discharges," IEEE Transactions on Dielectrics and Electrical Insulation, vol. 19, no. 6, pp. 2189-2195, Dec. 2012.

81. C. Naresh, R. Jayaganthan, R. Velmurugan, and R. Sarathi, "Investigation on the digital image correlation and charge trap characteristics of Al/epoxy nanocomposites," Materials Research Express, vol. 7, no. 2, p. 025035, Feb. 2020.

82. C. Naresh, R. Jayaganthan, B. Nageshwar Rao, M. Danikas, T. Tanaka, and R. Sarathi, "Investigation on space charge and charge trap characteristics of Al-epoxy nanocomposites," IET Science, Measurement \& Technology, vol. 14, no. 2, pp. 146-156, Mar. 2020.

83. K. J. Kao, S. S. Bamji, and M. M. Perlman, "Thermally stimulated discharge current study of surface charge release in polyethylene by corona-generated excited molecules, and the crossover phenomenon," Journal of Applied Physics, vol. 50, no. 12, pp. 81818185, Dec. 1979.

84. M. Geiger et al., "Quantitative analysis of the density of trap states in semiconductors by electrical transport measurements on lowvoltage field-effect transistors.," Physical Review Applied, vol. 10, no. 4, p. 044023 , Oct. 2018.

85. D. Saha, A. G. Anisimov, R. M. Groves, I. A. Tsekmes, P. H. F. Morshuis, and R. Kochetov, "Epoxy-hBN nanocomposites: A study on space charge behavior and effects upon material," IEEE Transactions on Dielectrics and Electrical Insulation, vol. 24, no. 3, pp. 1718-1725, Jun. 2017.

86. P. Molinie, S. Agnel, and J. Castellon, "Investigations on charge accumulation and relaxation in polycrystalline A12O3: Correlations of surface potential measurements with other techniques," in 2010 10th IEEE International Conference on Solid Dielectrics, 2010, pp. $1-4$.

87. M. A. Noras, "Non-contact surface charge / voltage measurements. Fieldmeter and voltmeter methods," Trek Application note, no. 3002, p. 6, 2002 .

88. D. K. Das-Gupta, "Decay of electrical charges on organic synthetic polymer surfaces," IEEE Transactions on Electrical Insulation, vol. 25, no. 3, pp. 503-508, Jun. 1990

89. E. A. Baum, T. J. Lewis, and R. Toomer, "The lateral motion of charge on thin films of polyethylene terephthalate," Journal of Physics D: Applied Physics, vol. 11, no. 6, pp. 963-977, Apr. 1978.

90. A. Crisci, B. Gosse, J.-P. Gosse, and V. Ollier-Duréault, "Surfacepotential decay due to surface conduction," The European Physical Journal Applied Physics, vol. 4, no. 1, pp. 107-116, Oct. 1998.

91. H. Sjostedt, S. Gubanski, and Y. Serdyuk, "Charging Characteristics of EPDM and Silicone Rubbers Deduced from Surface Potential Measurements," IEEE Transactions on Dielectrics and Electrical Insulation, vol. 16, no. 3, pp. 696-703, Jun. 2009.

92. J. Kindersberger and C. Lederle, "Surface charge decay on insulators in air and sulfurhexafluorid - part I: simulation," IEEE Transactions on Dielectrics and Electrical Insulation, vol. 15, no. 4, pp. 941-948, Aug. 2008

93. R. Coelho, L. Levy, and D. Sarrail, "On the Natural Decay of Corona Charged Insulating Sheets," physica status solidi (a), vol. 94, no. 1, pp. 289-298, Mar. 1986.

94. A. Neves and H. J. A. Martins, "Surface charging and charge decay in solid dielectrics," in Conference Record of IEEE International Symposium on Electrical Insulation, 1996, vol. 2, pp. 782-786.

95. J. R. S. S. Kumara, Y. V. Serdyuk, and S. M. Gubanski, "Surface potential decay on LDPE and LDPE/A12O3 nanocomposites: Measurements and modeling," IEEE Transactions on Dielectrics and Electrical Insulation, vol. 23, no. 6, pp. 3466-3475, Dec. 2016.

96. Y. S. Kwon, A. A. Gromov, A. P. Ilyin, and G.-H. Rim, "Passivation process for superfine aluminum powders obtained by electrical explosion of wires," Applied Surface Science, vol. 211, no. 1-4, pp. 57-67, Apr. 2003.

97. W. Zhou and D. Yu, "Fabrication, thermal, and dielectric properties of self-passivated Al/epoxy nanocomposites," Journal of Materials Science, vol. 48, no. 22, pp. 7960-7968, Nov. 2013.

98. S. Paul and T. K. Sindhu, "Effect of filler particle size on electric energy density of epoxy-aluminum nanocomposites," IEEE Transactions on Dielectrics and Electrical Insulation, vol. 23, no. 5, pp. 2786-2794, Oct. 2016.

99. X. Y. Huang, P. K. Jiang, and C. U. Kim, "Electrical properties of polyethylene/aluminum nanocomposites," Journal of Applied Physics, vol. 102, no. 12, p. 124103, Dec. 2007.

100. Z. Wang, W. Zhou, X. Sui, and L. Dong, "Enhanced dielectric properties and thermal conductivity of $\mathrm{Al} / \mathrm{CNTs} / \mathrm{PVDF}$ ternary composites," Journal of Reinforced Plastics and Composites, vol. 34, no. 14, pp. 1126-1135, Jul. 2015.

101. C. Naresh, R. Sarathi, and R. Jayaganthan, "Effect of Interphase on the Space Charge Performance of Al-Epoxy Nanocomposites," in International Symposium on Electrical Insulating Materials (ISEIM), 2020, pp. 387-390.

102. C. Naresh, R. Sarathi, and R. Jayaganthan, "Impact of gammairradiation on space charge and charge trap characteristics of Al/epoxy nanocomposites," SPE Polymers, vol. 2, no. 1, pp. 38-49, Feb. 2021.

103. B. X. Du, J. P. Jiang, J. G. Zhang, and D. S. Liu, "Dynamic behavior of surface charge on double-layer oil-paper insulation under pulse voltage," IEEE Transactions on Dielectrics and Electrical Insulation, vol. 23, no. 5, pp. 2712-2719, Oct. 2016.

04. J. Chen and J. H. Davidson, "Model of the Negative DC Corona Plasma: Comparison to the Positive DC Corona Plasma," Plasma Chemistry and Plasma Processing, vol. 23, no. 1, pp. 83-102, 2003.

105. B. X. Du, J. Li, W. Du, H. Du, Y. Gao, and L. Yang, "Dynamic behavior of surface charge on direct-fluorinated polyimide films," in 2013 IEEE International Conference on Solid Dielectrics (ICSD), 2013, pp. 329-332.

106. B. M. A. Desai, P. Mishra, N. J. Vasa, R. Sarathi, and T. Imai, "Understanding the performance of corona aged epoxy nano micro composites," Micro \& Nano Letters, vol. 13, no. 9, pp. 1280-1285, Sep. 2018.

107. S. Raetzke and J. Kindersberger, "Role of interphase on the resistance to high-voltage arcing, on tracking and erosion of silicone/SiO 2 nanocomposites," IEEE Transactions on Dielectrics and Electrical Insulation, vol. 17, no. 2, pp. 607-614, Apr. 2010.

108. T. Tanaka, M. Kozako, N. Fuse, and Y. Ohki, "Proposal of a multicore model for polymer nanocomposite dielectrics," IEEE Transactions on Dielectrics and Electrical Insulation, vol. 12, no. 4, pp. 669-681, Aug. 2005.

109. N. Longiéras, M. Sebban, P. Palmas, A. Rivaton, and J. L. Gardette, "Degradation of epoxy resins under high energy electron beam irradiation: Radio-oxidation," Polymer Degradation and Stability, vol. 92, no. 12, pp. 2190-2197, Dec. 2007.

110. G. Chen, A. E. Davies, and H. M. Banford, "Influence of radiation environments on space charge formation in $\gamma$-irradiated LDPE," IEEE Transactions on Dielectrics and Electrical Insulation, vol. 6, no. 6, pp. 882-886, 1999.

111. Y. Gao and B. X. Du, "Effect of gamma-ray irradiation on surface charge decay through bulk of epoxy resin," in 2012 IEEE International Symposium on Electrical Insulation, 2012, vol. 27, no. 12, pp. 531-534.

$\begin{array}{ll}\text { kGy } & \text { kilo gray } \\ \mathrm{LN}_{2} & \text { Liquid nitrogen } \\ \mathrm{UV} & \text { Ultra violet }\end{array}$

\section{Variables}

A Scan area

d Gap distance between probe and sample surface

$\Delta \mathrm{E} \quad$ Trap depth

$\mathrm{E}_{\mathrm{c}} \quad$ Energy levels of conduction

$\mathrm{E}_{\mathrm{d}} \quad$ Demarcation energy 
Chillu Naresh, Rengaswamy Jayaganthan, Ramanujam Sarathi and Michael G. Danikas/

Journal of Engineering Science and Technology Review 14 (3) (2021) 179 - 193

$\mathrm{I}_{\mathrm{d}} \quad$ Detrapping emission current

k Boltzmann constant

L Thickness of the material

$v \quad$ Attempt to escape frequency

N(E) Density of charges at an energy level E

Q Charge on the test surface

$\sigma \quad$ Surface charge density over the material surface

$\mathrm{T}$ Thermodynamic temperature

$t_{f} \quad$ Front time

V Surface potential

$\mathrm{V}_{0} \quad$ Initial surface potential

$\varepsilon \quad$ Permittivity of the material between electrodes

$\varepsilon_{\mathrm{r}} \quad$ Relative permittivity of the material

$\lambda \quad$ Decay rate constant

$\tau \quad$ Mean lifetime of the carriers 